\title{
Strict Confluent Drawing
}

\author{
David Eppstein ${ }^{1}$, Danny Holten ${ }^{2}$, Maarten Löffler ${ }^{3}$, \\ Martin Nöllenburg ${ }^{4}$, Bettina Speckmann ${ }^{5}$, and Kevin Verbeek ${ }^{6}$ \\ 1 Computer Science Department, University of California, Irvine, USA \\ eppstein@uci.edu \\ 2 Synerscope BV, Eindhoven, the Netherlands \\ danny.holten@synerscope.com
}

3 Department of Computing and Information Sciences, Utrecht University, The Netherlands

m.loffler@uu.nl

4 Institute of Theoretical Informatics, Karlsruhe Institute of Technology, Germany

noellenburg@kit.edu

5 Department of Mathematics and Computer Science,

Technical University Eindhoven, The Netherlands

speckman@win.tue.nl

${ }^{6}$ Department of Computer Science, University of California, Santa Barbara, USA

kverbeek@cs.ucsb.edu

\begin{abstract}
We define strict confluent drawing, a form of confluent drawing in which the existence of an edge is indicated by the presence of a smooth path through a system of arcs and junctions (without crossings), and in which such a path, if it exists, must be unique. We prove that it is NP-complete to determine whether a given graph has a strict confluent drawing but polynomial to determine whether it has an outerplanar strict confluent drawing with a fixed vertex ordering (a drawing within a disk, with the vertices placed in a given order on the boundary).
\end{abstract}

\section{Introduction}

Confluent drawing is a style of graph drawing in which edges are not drawn explicitly; instead vertex adjacency is indicated by the existence of a smooth path through a system of arcs and junctions that resemble train tracks. These types of drawings allow even very dense graphs, such as complete graphs and complete bipartite graphs, to be drawn in a planar way [4]. Since its introduction, there has been much subsequent work on confluent drawing [7]6]9|10|13|17], but the complexity of confluent drawing has remained unclear: how difficult is it to determine whether a given graph has a confluent drawing? Confluent drawings have a certain visual similarity to a graph drawing technique called edge bundling [3 5 11 12 14], in which "similar" edges are routed together in "bundles", but we note that these drawings should be interpreted differently. In particular, sets of edges bundled together form visual junctions, however, interpreting them as confluent junctions can create false adjacencies.

Formally, a confluent drawing may be defined as a collection of vertices, junctions and arcs in the plane, such that all arcs are smooth and start and end at either a junction or a vertex, such that arcs intersect only at their endpoints, and such that all arcs that meet at a junction share the same tangent line there. A confluent drawing $D$ represents a graph $G$ defined as follows: the vertices of $G$ are the vertices of $D$, and there is an

S. Wismath and A. Wolff (Eds.): GD 2013, LNCS 8242, pp. 352-363, 2013.

(C) Springer International Publishing Switzerland 2013 
edge between two vertices $u$ and $v$ if and only if there exists a smooth path in $D$ from $u$ to $v$ that does not pass any other vertex. (In some variants of confluent drawing an additional restriction is made that the smooth path may not intersect itself [13]; however, this constraint is not relevant for our work.)

Contribution. In this paper we introduce a subclass of confluent drawings, which we call strict confluent drawings. Strict confluent drawings are confluent drawings with the additional restrictions that between any pair of vertices there can be at most one smooth path, and there cannot be any paths from a vertex to itself. Figure 1 illustrates the forbidden configurations. To avoid irrelevant components in the drawing, we also require all arcs of the drawing to be part of at least one smooth path representing an edge. We

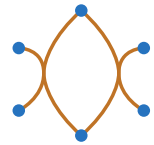

(a)

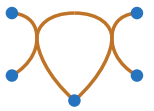

(b)

Fig. 1. (a) A drawing with a duplicate path. (b) A drawing with a self-loop. believe that these restrictions may make strict drawings easier to read, by reducing the ambiguity caused by the existence of multiple paths between vertices. In addition, as we show, the assumption of strictness allows us to completely characterize their complexity, the first such characterization for any form of confluence on arbitrary undirected graphs.

We prove the following:

- It is NP-complete to determine whether a given graph has a strict confluent drawing.

- For a given graph, with a given cyclic ordering of its vertices, there is a polynomial time algorithm to find an outerplanar strict confluent drawing, if it exists: this is a drawing in a disk, with the vertices in the given order on the boundary of the disk

- When a graph has an outerplanar strict confluent drawing, an algorithm based on circle packing can construct a layout of the drawing in which every arc is drawn using at most two circular arcs.

See Fig.2(a) for an example of an outerplanar strict confluent drawing. Previous work on tree-confluent [13] and delta-confluent drawings [6] characterized special cases of outerplanar strict confluent drawings as being the chordal bipartite graphs and distancehereditary graphs respectively, so these graphs as well as the outerplanar graphs are all outerplanar strict confluent. The six-vertex wheel graph in Fig.2(b) provides an example

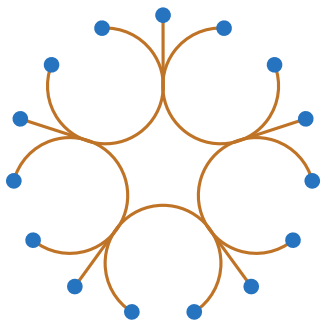

(a)

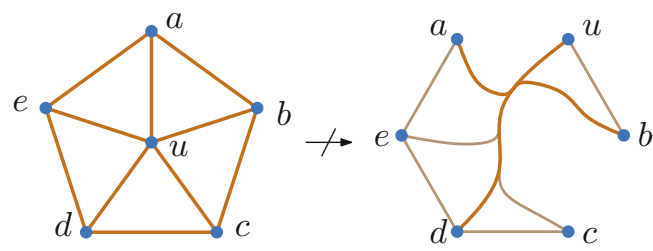

(b)

Fig. 2. (a) Outerplanar strict confluent drawing of the GD2011 contest graph. (b) A graph with no outerplanar strict confluent drawing. 
of a graph that does not have an outerplanar strict confluent drawing. (The central vertex $u$ needs to be placed between two of the outer vertices, say, $a$ and $b$. The smooth path from $u$ to the opposite vertex $d$ separates $a$ and $b$, so there must be a junction shared by the $u-d$ and $a-b$ paths, creating a wrong adjacency with $d$.)

\section{Preliminaries}

Let $G=(V, E)$ be a graph. We call an edge $e$ in a drawing $D$ direct if it consists only of a single arc (that does not pass through junctions). We call the angle between two consecutive arcs at a junction or vertex sharp if the two arcs do not form a smooth path; each junction has exactly two angles that are not sharp, and every angle at a vertex is sharp (so the number of sharp angles equals the degree of the vertex).

Lemma 1. Let $G$ be a graph, and let $E^{\prime} \subseteq E$ be the edges of $E$ that are incident to at least one vertex of degree 2. If $G$ has a strict confluent drawing $D$, then it also has a strict confluent drawing $D^{\prime}$ in which all edges in $E^{\prime}$ are direct.

Proof. Let $v$ be a degree- 2 vertex in $G$ with two incident edges $e$ and $f$. We consider the representation of $e$ and $f$ in $D$ and modify $D$ so that $e$ and $f$ are single arcs. There are two cases. If $e$ and $f$ leave $v$ on two disjoint paths, then these paths have only merge junctions from $v$ 's perspective. We can simply separate these junctions from $e$ and $f$ as shown in Fig. 3(a) If, on the other hand, $e$ and $f$ share the same path leaving $v$, then their paths split at some point. We need to reroute the merge junctions prior to the split and separate the merge junctions after the split as shown in Fig. 3(b). This is always possible since $v$ has no other incident edges. Because $D$ was strict and these changes do not affect strictness, $D^{\prime}$ is still a strict confluent drawing and edges $e$ and $f$ are direct.

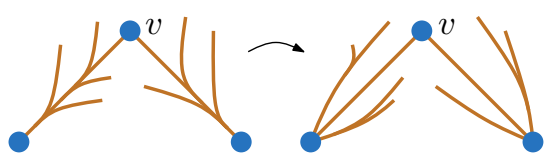

(a)

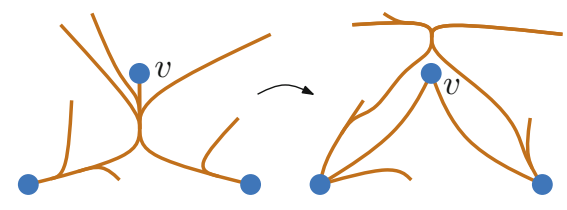

(b)

Fig. 3. The two cases of creating single arcs for edges incident to a degree- 2 vertex

Lemma 2. Let $G$ be a graph. If $G$ has no $K_{2,2}$ as a subgraph, whose vertices have degrees $\geq 3$ in $G$, then $G$ has a strict confluent drawing if and only if $G$ is planar.

Proof. Since every planar drawing is also a strict confluent drawing, that implication is obvious. So let $D$ be a strict confluent drawing for a graph $G$ without a $K_{2,2}$ subgraph, whose vertices have degrees $\geq 3$ in $G$. Since larger junctions, where more than three arcs meet, can easily be transformed into an equivalent sequence of binary junctions, we can assume that every junction in $D$ is binary, i.e., two arcs merge into one (or, from 
a different perspective, one arc splits into two). By Lemma1 we can further transform $D$ so that all edges incident to degree- 2 vertices are direct. Now for any vertex $u$ in $D$ none of its outgoing paths to some neighbor $v$ can visit a merge junction before visiting a split junction as this would imply either a non-strict drawing or a $K_{2,2}$ subgraph with vertex degrees $\geq 3$. So the sequence of junctions on any $u-v$ path consists of a number of split junctions followed by a number of merge junctions. But any such path can be unbundled from its junctions to the left and right and turned into a direct edge without creating arc intersections as illustrated in Fig. 4. This shows that $D$ can be transformed into a standard planar drawing of $G$.

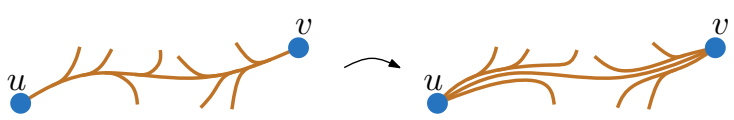

Fig. 4. Any strict confluent drawing of a graph without a $K_{2,2}$ subgraph can be transformed into a standard planar drawing

Lemma 3 characterizes the combinatorial complexity of strict confluent drawings. Its proof is found in the full paper [8] and uses Euler's formula and double counting.

Lemma 3. The combinatorial complexity of any strict confluent drawing $D$ of a graph $G$, i.e., the number of arcs, junctions, and faces in $D$, is linear in the number of vertices of $G$.

Lemma 3 is in contrast to previous methods for confluently drawing interval graphs [4] and for drawing confluent Hasse diagrams [9], both of which may produce (non-strict) drawings with quadratically many features.

\section{Computational Complexity}

We will show by a reduction from planar 3-SAT [15] that it is NP-complete to decide whether a graph $G$ has a strict confluent drawing in which all edges incident to degree-2 vertices are direct. By Lemma1, this is enough to show that it is also NP-complete to decide if $G$ has any strict confluent drawing.

Consider the subdivided grid graph (a grid with one extra vertex on each edge). In this graph, all edges are adjacent to a degree 2 vertex. Since a grid graph more than one square wide has only one fixed planar embedding (up to choice of the outer face), the subdivided grid graph has only one confluent embedding in which all edges are direct. We will base our construction on a number of such grids.

Let $S$ be a planar 3-SAT formula. Globally speaking, we will create a grid graph for each variable of $S$, of size depending on the number of clauses that the variable appears in. The external edges of this grid graph are alternatingly colored green and red. We connect the variable graphs by identifying certain vertices: for each of the three variables that appear in a clause, we select one subdivided edge (that is, three vertices connected by two edges) on the outer face, and identify the endpoints of these edges 


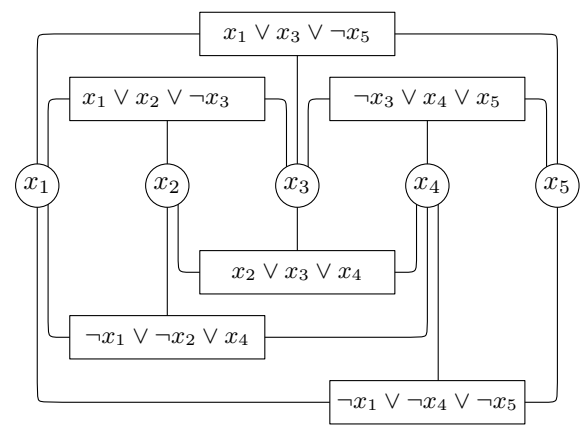

(a)

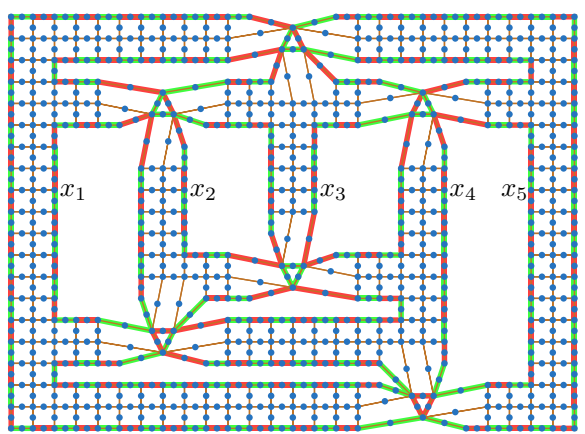

(b)

Fig. 5. (a) A planar 3-SAT formula. (b) The corresponding global frame of the construction: one grid graph per variable, with some vertices identified at each clause. Green boundary edges correspond to positive literals, red edges to negated literals. For easier readability the grids in this figure are larger than strictly necessary.
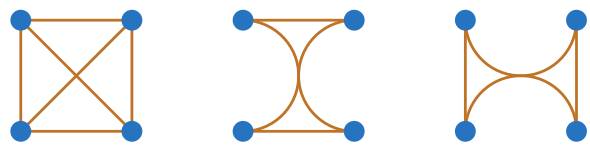

Fig. 6. $K_{4}$ and its two strict confluent drawings, without moving the vertices and keeping all arcs inside the convex hull of the vertices

into a triangle of subdivided edges (that is, a 6 -cycle). We choose a green edge for a positive occurrence of the variable and a red edge for a negated occurrence. This will become clear below. We call the resulting graph $F$ the frame of the construction; all edges of $F$ are adjacent to a degree- 2 vertex and $F$ has only one planar embedding (up to choice of the outer face). Figure 5 shows an example.

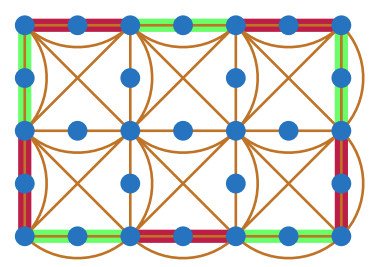

(a)

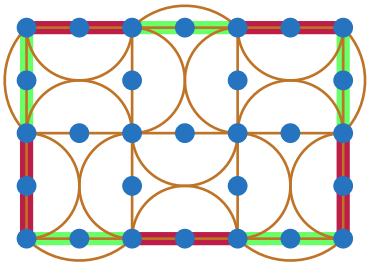

(b)

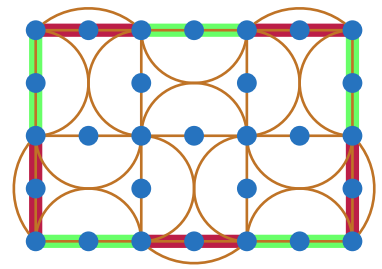

(c)

Fig. 7. (a) A variable gadget consists of a grid of $K_{4}$ 's. Green (light) edges of the frame highlight normal literals, red (dark) edges negated ones. (b) One of the two possible strict confluent drawings, corresponding to the value true. (c) The other strict confluent drawing, corresponding to false. 
The main idea of the construction is based on the fact that $K_{4}$, when drawn with all four vertices on the outer face, has exactly two strict confluent drawings: we need to create a junction that merges the diagonal edges with one pair of opposite edges, and we can choose the pair. Figure 6 illustrates this. We will add a copy of $K_{4}$ to every cell of the frame graph $F$. Recall that every cell, except for the triangular clause faces, is a subdivided square (that is, an 8-cycle). We add $K_{4}$ on the four grid vertices (not the subdivision vertices). The edges that connect external grid vertices are called literal edges. Figure 7(a) shows this for a small grid. Since neighboring grid cells share a (subdivided) edge, the $K_{4}$ 's are not edgeindependent. This implies that in a strict confluent drawing, we cannot "use" such a common edge in

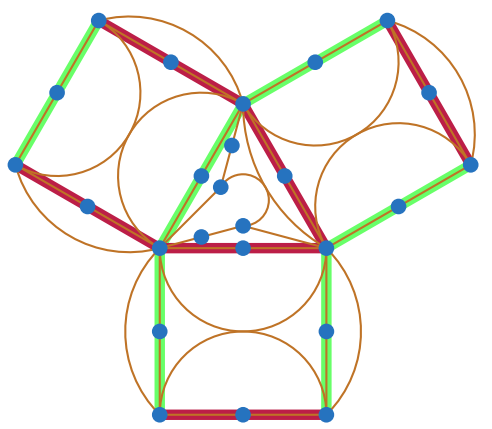

Fig. 8. Three variables attached to a clause gadget. The top left variable occurs in the clause as a positive literal, the others as negative literals. The clause can be satisfied because the top right variable is set to false. both cells. Therefore, we need to orient the $K_{4^{-}}$ junctions alternatingly, as illustrated in Figures $7(\mathrm{~b})$ and $7(\mathrm{c})$, If the grid is sufficiently large (every cell is part of a larger at least size- $(2 \times 2)$ grid) these choices are completely propagated through the entire grid, so there are two structurally different possible embeddings, which we use to represent the values true and false of the corresponding variable. For every green edge of the frame in the true state and every red edge in the false state there is one remaining literal edge in the outer face, which can still be drawn either inside or outside their grid cells. In the opposite states these literal edges are needed inside the grid cells to create the $K_{4}$ junctions. The availability of at least one literal edge (corresponding to a true literal) is important for satisfying the clause gadgets, which we describe next.

Inside each triangular clause face, we add the graph depicted in Figure 9(a). This graph has several strict confluent drawings; however, in every drawing at least one of the three outer edges needs to be drawn inside the subdivided triangle.

Lemma 4. There is no strict confluent drawing of the clause graph in which all three long edges are drawn outside. Moreover, there is a strict confluent drawing of the clause graph with two of these edges outside, for every pair.

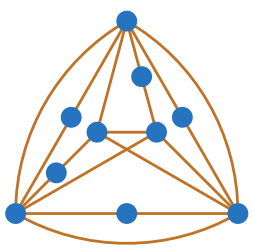

(a)

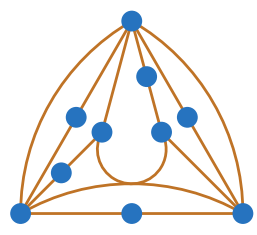

(b)

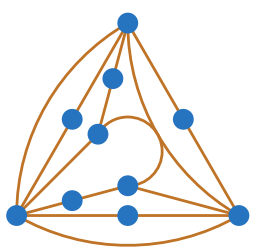

(c)

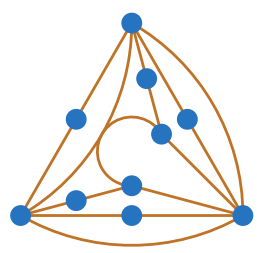

(d)

Fig. 9. (a) The input graph of the clause. (b, c, d) Three different strict confluent drawings 
Proof. Recall that by Lemma 1 the subdivided triangle must be embedded as a 6 -cycle of direct arcs. To prove the first part of the lemma, assume that the triangle edges are all drawn outside this cycle. The remainder of the graph has no 4-cycles without subdivision vertices (that is, no $K_{2,2}$ with higher-degree vertices), so by Lemma 2 it can only have a strict confluent drawing if it is planar. However, it is a subdivided $K_{5}$, which is not planar. To prove the second part of the lemma, we refer to Figures $9(\mathrm{~b}), 9(\mathrm{c})$ and $9(\mathrm{~d})$.

This describes the reduction from a planar 3-SAT instance to a graph consisting of variable and clause gadgets. Next we show that this graph has a strict confluent drawing if and only if the planar 3-SAT formula is satisfiable. For a given satisfying assignment we choose the corresponding embeddings of all variable gadgets. The assignment has at least one true literal per clause, and correspondingly in each clause gadget one of the three literal edges can be drawn inside the clause triangle, allowing a strict confluent drawing by Lemma 4 . Conversely, in any strict confluent drawing, each clause must be drawn with at least one literal edge inside the clause triangle by Lemma 4, so translating the state of each variable gadget into its truth value yields a satisfying assignment.

To show that testing strict confluence is in NP, recall that by Lemma 3 the combinatorial complexity of the drawing is linear in the number of vertices. Thus the existence of a drawing can be verified by guessing its combinatorial structure and verifying that it is planar and a drawing of the correct graph.

Theorem 1. Deciding whether a graph has a strict confluent drawing is NP-complete.

\section{Outerplanar Strict Confluent Drawings}

For a graph $G$ with a fixed cyclic ordering of its vertices, we can test in polynomial time whether an outerplanar strict confluent drawing with this vertex ordering exists, and, if so, construct one. This algorithm uses the closely related notion of a canonical diagram of $G$, which is unique and exists if and only if an outerplanar strict confluent drawing exists. From the canonical diagram a confluent drawing can be constructed. We further show that the drawing can be constructed such that every arc consists of at most two circular arcs.

\subsection{Canonical Diagrams}

We define a canonical diagram to be a collection of junctions and arcs connecting the vertices in the given order on the outer face (as in a confluent drawing), but with some of the faces of the diagram marked, satisfying additional constraints enumerated below. Figure 10 shows a canonical diagram and an outerplanar strict confluent drawing of the same graph. In such a diagram, a trail is a smooth curve from one vertex to another that follows the arcs (as in a confluent drawing) but is allowed to cross the interior of marked faces from one of its sharp corners to another. The constraints are:

- Every arc is part of at least one trail.

- No two trails between the same two vertices can follow different sequences of arcs and faces. 

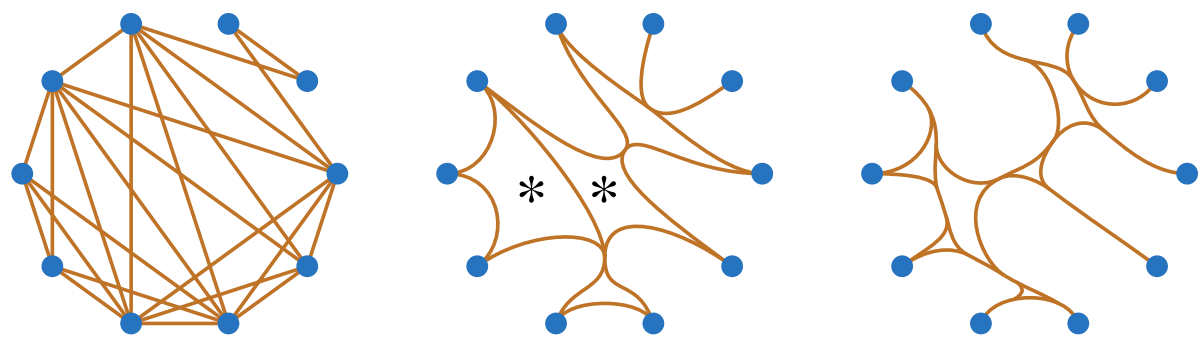

Fig. 10. Three views of the same graph as a node-link diagram (left), canonical diagram (center), and outerplanar strict confluent drawing (right)

- Each marked face must have at least four angles, all of which are sharp.

- Each arc must have either sharp angles or vertices at both of its ends.

- For each junction $j$ with exactly two arcs in each direction, let $f$ and $f^{\prime}$ be the two faces with sharp angles at $j$. Then it is not allowed for $f$ and $f^{\prime}$ to both be either marked or to be a triangle (a face with three angles, all sharp).

Let $j$ be a junction of a canonical diagram $D$. Then define the funnel of $j$ to be the 4-tuple of vertices $a, b, c, d$ where $a$ is the vertex reached by a path that leaves $j$ in one direction and continues as far clockwise as possible, $b$ is the most counterclockwise vertex reachable in the same direction from $j, c$ is the most clockwise vertex reachable in the other direction, and $d$ is the most counterclockwise vertex reachable in the other direction. Note that none of the paths from $j$ to $a, b, c$, and $d$ can intersect each other without contradicting the uniqueness of trails. We call the circular intervals of vertices $[a, b]$ and $[c, d]$ (in the counterclockwise direction) the funnel intervals of the respective funnel. We say a circular interval $[a, b]$ is separated if either $a$ and $b$ are not adjacent in $G$, or there exists a junction in the canonical diagram with funnel intervals $[a, e]$ and $[f, b]$, where $e, f \in[a, b]$.

A canonical diagram represents a graph $G$ in which the edges in $G$ correspond to trails in the diagram. As we show in the full paper [8], a graph $G$ has a canonical diagram if and only if it has an outerplanar strict confluent drawing, and if a canonical diagram exists then it is unique.

\subsection{Algorithm}

By using the properties of canonical diagrams (see the full paper [8]), we may obtain an algorithm that constructs a canonical diagram and strict confluent drawing of a given cyclically-ordered graph $G$, or reports that no drawing exists, in time and space $O\left(n^{2}\right)$. This bound is optimal in the worst case, as it matches the input size of a graph that may have quadratically many edges.

Steps 1-3 of the algorithm, detailed below, build some simple data structures that speed up the subsequent computations. Step 4 discovers all of the funnels in the input, from which it constructs a list of all of the junctions of the canonical diagram. Step 5 connects these junctions into a planar drawing, a subset of the canonical diagram. Step 6 
builds a graph for each face of this drawing that will be used to complete it into the entire canonical diagram, and step 7 uses these graphs to find the remaining arcs of the diagram and to determine which faces of the diagram are marked. Step 8 checks that the diagram constructed by the previous steps correctly represents the input graph, and step 9 splits the marked faces, converting the diagram into a strict confluent drawing.

1. Number the vertices clockwise around the boundary cycle from 0 to $n-1$.

2. Build a table, containing for each pair $i, j$, the number of ordered pairs $\left(i^{\prime}, j^{\prime}\right)$ with $i^{\prime} \leq i, j^{\prime} \leq j$, and vertices $i^{\prime}$ and $j^{\prime}$ adjacent in $G$. By performing a constant number of lookups in this table we may determine in constant time how many edges exist between any two disjoint intervals of the boundary cycle.

3. Build a table that lists, for each ordered pair $u, v$ of vertices, the neighbor $w$ of $u$ that is closest in clockwise order to $v$. That is, $w$ is adjacent to $u$, and the interval from $v$ clockwise to $w$ contains no other neighbors of $u$. The table entries for $u$ can be found in linear time by a single counterclockwise scan. Repeat the same construction in the opposite orientation.

4. For each separated interval $[a, b]$, let $c$ be the next neighbor of $a$ that is counterclockwise of $b$, and let $d$ be the next neighbor of $b$ that is clockwise of $a$. If (i) $c$ is a neighbor of $b$, (ii) $d$ is a neighbor of $a$, (iii) $a$ is the next neighbor of $c$ that is counterclockwise of $d$, and (iv) $b$ is the next neighbor of $d$ that is clockwise of $c$, then (if a confluent diagram exists) $a, b, c, d$ must form the funnel of a junction, and all funnels have this form. We check all circular intervals in increasing order of their cardinalities. For each discovered funnel, we mark the intervals that are separated by the corresponding junction. This way we can check in $O(1)$ time whether a circular interval is separated. If the number of funnels exceeds the linear bound of Lemma 3 on the number of junctions in a confluent drawing, abort the algorithm.

5. Create a junction for each of the funnels found in step 4 . For each vertex $v$, make a set $J_{v}$ of the junctions whose funnel includes that vertex; if they are to be drawn as part of a canonical diagram, the junctions of $J_{v}$ need to be connected to $v$ by a confluent tree. For any two junctions in $J_{v}$, it is possible to determine in constant time whether one is an ancestor of another in this tree, or if not whether one is clockwise of the other, by examining the cyclic ordering of vertices in their funnels. Construct the trees of junctions and their planar embedding in this way. The result of this stage of the algorithm should be a planar embedding of part of the canonical diagram consisting of all vertices and junctions, and the subset of the arcs that are part of a path from a junction to one of its funnel vertices. Check that the embedding is planar by computing its Euler characteristic, and abort the algorithm if it is not.

6. For each face $f$ of the drawing created in step 5, and each pair $j, j^{\prime}$ of junctions belonging to $f$, use the data structure from step 2 to test whether there is an edge whose trail passes through both $j$ and $j^{\prime}$. This results in a graph $H_{f}$ in which the vertices represent the vertices or junctions on the boundary of $f$ and the edges represent pairs of vertices or junctions that must be connected, either by an arc or by shared membership in a marked face. The remaining arcs to be drawn in $f$ will be exactly the edges of $H_{f}$ that are not crossed by other edges of $H_{f}$; the marked faces in $f$ will be exactly the faces that contain pairs of crossing edges of $H_{f}$.

7. Within each face $f$ of the drawing so far, build a table using the same construction as in step 2 that can be used to determine the existence of a crossing edge for an 
edge in $H_{f}$ in constant time. Use this data structure to identify the crossed edges, and draw an arc in $f$ for each uncrossed edge. For each face $g$ of the resulting subdivision of $f$, if $g$ has four or more vertices or junctions, find two pairs that would cross and test whether both pairs correspond to edges in $H_{f}$; if so, mark $g$.

8. Construct a directed graph that has a vertex for each vertex of $G$, two vertices for each junction of the diagram (one in each direction), two directed edges for each arc, and a directed edge for each ordered pair of sharp angles that are non-consecutive in a marked face. By performing a depth-first search in this graph, determine whether there exist multiple smooth paths in the resulting drawing from any vertex of $G$ to any other point in the drawing, and abort the algorithm if any such pair of paths is found. Determine the set of vertices of $G$ reachable from $v$ and verify that it is the same set of vertices that are reachable in the original graph. Additionally, verify that the diagram satisfies the requirements in the definition of a canonical diagram. Abort the algorithm if any inconsistency is found in this step.

9. Convert the canonical diagram into a confluent drawing and return it.

Theorem 2. For a given n-vertex graph $G$, and a given circular ordering of its vertices, it is possible to determine whether $G$ has an outerplanar strict confluent drawing with the given vertex ordering, and if so to construct one, in time $O\left(n^{2}\right)$.

\subsection{Drawings with Low Curve Complexity}

Suppose that we are given a topological description of an outerplanar strict confluent drawing $D$ of a connected graph $G$, describing the tangency pattern and ordering of the arcs at each junction. It still remains to draw $D$ (or possibly an equivalent but combinatorially different outerplanar strict confluent drawing) in the plane using concrete curves for its arcs. If we ignore the tangency requirements at its junctions, the arcs and junctions of $D$ form a planar graph, but applying standard planar graph drawing methods will generate arcs that may not be smooth and that are not tangent to each other at the junctions. So how are we to draw $D$ ? Here we use a circle packing method to draw $D$ with a small number of circular arcs for each arc of $D$. Thus, these drawings have low curve complexity in the sense of Bekos et al. [1], but with this complexity measured along arcs of the confluent diagram rather than edges of another type of graph drawing.

Given such a drawing $D$, let $D^{\prime}$ be a modified version of $D$ in which every junction is incident to exactly three arcs, formed from $D$ by suppressing two-arc junctions and splitting junctions with more than three arcs. Assume also (again by adding more junctions if necessary) that each vertex in $D^{\prime}$ has only a single arc incident to it.

Given the topological diagram $D^{\prime}$, we form a planar graph $H$ that has a vertex for each vertex or junction of $D^{\prime}$, and an edge for each arc of $D^{\prime}$. Additionally, we create an edge in $H$ for each two vertices that are consecutive in the cyclic ordering of the vertices around the disk containing the drawing.

Lemma 5. $H$ is planar, 3-regular, and 3-vertex-connected.

Proof. Planarity and 3-regularity follow immediately from the construction of $H$. Every two vertices of $G$ are connected by three vertex-disjoint paths in $H$ : at least one (not necessarily a smooth path) through $D$, using the assumption that $G$ is connected, and two 
more around the boundary of the disk. Therefore, if $H$ were not 3-vertex-connected, only one of its 3-connected components could contain vertices of $G$. The other components would either contain components of $D$ that are not part of any smooth path between vertices of $G$ (forbidden in a strict confluent drawing) or would contain more than one smooth path between the same sets of vertices (also forbidden).

Theorem 3. Let $D$ be an outerplanar strict confluent drawing of a graph $G$, given topologically but not geometrically. Then we can construct an outerplanar strict confluent drawing of $G$ in which each arc of the drawing is represented by a smooth curve that is either a circular arc or the union of two circular arcs.

Proof. By the Koebe-Thurston-Andreev circle packing theorem, there exists a system $C$ of circles representing the faces of $H$, such that two circles are adjacent exactly when the corresponding faces share an edge. We may assume (by performing a Möbius transformation if necessary) that the outer circle of this circle packing corresponds to the outer face of $H$. C may be found efficiently (although not in strongly polynomial time) by a numerical iteration that quickly converges to the system of radii of the circles, from which their centers can also be computed easily [2[16].

Each vertex of $G$ corresponds in $C$ to one of the triangular gaps between the outer circle and two other circles, and may be placed at the point of tangency of the two non-outer circles (one of the vertices of this triangle); see Fig.11. The junctions in $D^{\prime}$ lie at the meeting point of three faces of $H$, and correspond in $C$ to the remaining triangular gaps between three circles. A confluent drawing of $G$ may be formed by removing the outer circle, removing all circular arcs bounding the triangular gaps incident to the outer circle, and in each remaining triangular gap removing the arc that is on the other side of the sharp angle. The resulting drawing contracts some edges of $D^{\prime}$ to form junctions with four incident arcs, but this does not affect the correctness of the drawing. In the resulting drawing, arcs of the diagram that have merge points or vertices at both of their endpoints are drawn as

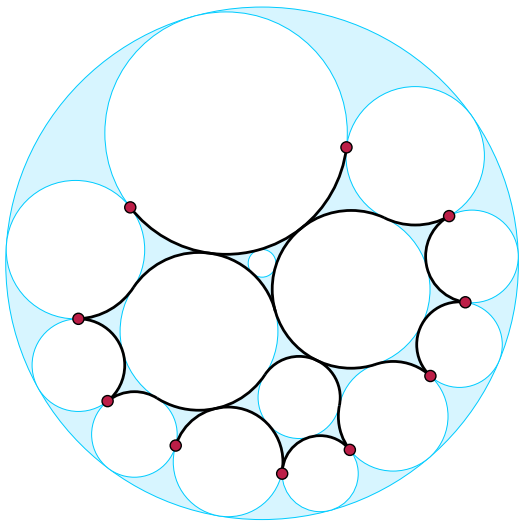

Fig. 11. Constructing an outerplanar strict confluent drawing from a circle packing. The vertices of the drawing correspond to triangular gaps adjacent to the outer circle, and the junctions to the remaining triangular gaps. two circular arcs (possibly both from the same circle); other arcs of the diagram are drawn as a single circular arc.

\section{Conclusions}

We have shown that, in confluent drawing, restricting attention to the strict drawings allows us to completely characterize their complexity, and we have also shown that outerplanar strict confluent drawings with a fixed vertex ordering may be constructed in 
polynomial time. The most pressing problem left open by this research is to recognize the graphs that have outerplanar strict confluent drawings, without imposing a fixed vertex order. Can we recognize these graphs in polynomial time?

Acknowledgements. This work originated at Dagstuhl seminar 13151, Drawing Graphs and Maps with Curves. D.E. was supported in part by the National Science Foundation under grants 0830403 and 1217322, and by the Office of Naval Research under MURI grant N00014-08-1-1015. M.L. was supported by the Netherlands Organisation for Scientific Research (NWO) under grant 639.021.123. M.N. received financial support by the 'Concept for the Future' of KIT under grant YIG 10-209.

\section{References}

1. Bekos, M.A., Kaufmann, M., Kobourov, S.G., Symvonis, A.: Smooth orthogonal layouts. In: Didimo, W., Patrignani, M. (eds.) GD 2012. LNCS, vol. 7704, pp. 150-161. Springer, Heidelberg (2013)

2. Collins, C.R., Stephenson, K.: A circle packing algorithm. Comput. Geom. Theory Appl. 25(3), 233-256 (2003)

3. Cui, W., Zhou, H., Qu, H., Wong, P.C., Li, X.: Geometry-based edge clustering for graph visualization. IEEE TVCG 14(6), 1277-1284 (2008)

4. Dickerson, M., Eppstein, D., Goodrich, M.T., Meng, J.Y.: Confluent drawings: Visualizing non-planar diagrams in a planar way. J. Graph. Algorithms Appl. 9(1), 31-52 (2005)

5. Dwyer, T., Marriott, K., Wybrow, M.: Integrating edge routing into force-directed layout. In: Kaufmann, M., Wagner, D. (eds.) GD 2006. LNCS, vol. 4372, pp. 8-19. Springer, Heidelberg (2007)

6. Eppstein, D., Goodrich, M.T., Meng, J.Y.: Delta-confluent drawings. In: Healy, P., Nikolov, N.S. (eds.) GD 2005. LNCS, vol. 3843, pp. 165-176. Springer, Heidelberg (2006)

7. Eppstein, D., Goodrich, M.T., Meng, J.Y.: Confluent layered drawings. Algorithmica 47(4), 439-452 (2007)

8. Eppstein, D., Holten, D., Löffler, M., Nöllenburg, M., Speckmann, B., Verbeek, K.: Strict confluent drawing. CoRR, abs/1308.6824 (2013)

9. Eppstein, D., Simons, J.A.: Confluent hasse diagrams. In: Speckmann, B. (ed.) GD 2011. LNCS, vol. 7034, pp. 2-13. Springer, Heidelberg (2011)

10. Hirsch, M., Meijer, H., Rappaport, D.: Biclique edge cover graphs and confluent drawings. In: Kaufmann, M., Wagner, D. (eds.) GD 2006. LNCS, vol. 4372, pp. 405-416. Springer, Heidelberg (2007)

11. Holten, D.: Hierarchical edge bundles: visualization of adjacency relations in hierarchical data. IEEE TVCG 12(5), 741-748 (2006)

12. Holten, D., van Wijk, J.J.: Force-Directed Edge Bundling for Graph Visualization. Computer Graphics Forum 28(3), 983-990 (2009)

13. Hui, P., Pelsmajer, M.J., Schaefer, M., Štefankovič, D.: Train tracks and confluent drawings. Algorithmica 47(4), 465-479 (2007)

14. Hurter, C., Ersoy, O., Telea, A.: Graph Bundling by Kernel Density Estimation. Computer Graphics Forum 31(3pt. 1), 865-874 (2012)

15. Lichtenstein, D.: Planar formulae and their uses. SIAM J. Comput. 11(2), 329-343 (1982)

16. Mohar, B.: A polynomial time circle packing algorithm. Discrete Math. 117(1-3), 257-263 (1993)

17. Quercini, G., Ancona, M.: Confluent drawing algorithms using rectangular dualization. In: Brandes, U., Cornelsen, S. (eds.) GD 2010. LNCS, vol. 6502, pp. 341-352. Springer, Heidelberg (2011) 